\title{
Radiation-Induced Synthesis of Polyvinylpyrrolidone (PVP) Nanogels
}

\author{
Adrian Alejandro Ges Naranjo ${ }^{1}$, HerlysViltres Cobas ${ }^{1,2}$, Danaydis Fonseca Rogdriguez ${ }^{3}$, Manuel Rapado \\ Paneque $^{4}$ and Yuri Aguilera Corrales ${ }^{1}$ \\ 1. Higher Institute of Technologies and Applied Science, Nuclear Development and Technological Applications Centre, La Habana, \\ Cuba \\ 2. Center of Research in Applied Science and Technology Advanced, Legaria-Unit, Polytechnic National Institute, Ciudad de Mexico, \\ Mexico \\ 3. Finlay Institute, La Habana, Cuba \\ 4. Center for Technological Applications and Nuclear Development, La Habana, Cuba
}

\begin{abstract}
Nanogels - particles of polymer gels having the dimensions in the order of nanometers - are gaining attention for their wide application as biomaterials. Mainly, the nanogels are promising novel pharmaceutical carriers for small biologically active agents, bio macromolecules and can be chemically modified to incorporate various ligands for targeted drug delivery. This important factor has stimulated research on dissimilar science field such as nanotechnology and biotechnology, polymer and materials sciences, biochemistry, radiation chemistry and pharmaceutical sciences. A multitude of techniques have been described for the synthesis of this nanomaterial from polymers. However, the use of ionizing radiation $(\gamma$, e-) has demonstrated to be especially suitable for obtaining polymeric nanogels with a high degree of purity for biomedical applications, although the gamma radiation has not been widely utilized for these purposes. The aim of this paper is to develop the synthesis of PVP (polyvynilpyrrolidone) nanogels by gamma irradiation, for their evaluation as potential pharmaceutical carriers. Experiments were performed using argon saturated solution of PVP $(0.1-1 \%)$. Crosslinking reactions were carried out in a gamma irradiation chamber with a ${ }^{60} \mathrm{Co}$ source (ISOGAMMA LLCo), at room temperature. The PVP concentration influence was evaluated in PVP solutions $(0.1 \%$ and $0.25 \%)$ at $15 \mathrm{kGy}$. The SEM (scanning electron microscopy), ATR (attenuate total reflection spectroscopy), DLS (dynamic light scattering), and viscosimetry were used as characterization techniques.
\end{abstract}

Key words: Polyvynilpyrrolidone nanogels, gamma irradiation, biomaterial.

\section{Introduction}

Soft nanomaterials (polymeric gels) have had a fast and brilliant career from an unwanted byproduct of polymerization processes. Also, it has converted in an important and fashionable topic of interdisciplinary research in the fields of polymer chemistry and physics, materials science, biochemistry, radiation chemistry and pharmaceutical sciences [1, 2]. Nano, micro and macro gels (most known in the form of water-swellable as hydrogels) have a multitude of

Corresponding author: Adrian Alejandro Ges Naranjo, research fields: radiation chemistry, polymer chemistry and nanotechnology. actual and potential applications, from filler materials in coating industry to modern biomaterials in pharmaceutical or environmental industry [3-9].

Nanogel is defined as the nanosized particles formed by physically or chemically crosslinked polymer networks, which is swell in a good solvent [10]. In other words, the Nano gelor a micro gel is an internally crosslinked macromolecule. This approach is based on the fact that, in principle, all the chain segments of a nanogel or micro gel are linked together, thus being a part of one macromolecule [1]. Therefore nanogels are similar to atypical single macromolecules in solution, but the presence of intramolecular bonds makes differences appear in its physicochemical 
properties, such as: surface/volume rate, shape and size, different rheological behavior, higher resistance to degradation and biocompatibility, high loading capacity of small biologically active agents and bio macromolecules within their structure, encapsulation or entrapment of drugs and stability of the resulting formulation in dispersion.

Natural polymers have not been widely used for this purpose since they vary in purity, and often require crosslinking that could denature the embedded drug. Consequently, synthetic polymers have received significantly more attention in this area [11], and particularly the hydrophilic polymers, thus capable of swelling in water. Typical hydrogel-forming polymers are those containing hydrophilic groups (i.e. $\mathrm{OH}$, $\mathrm{COOH}, \mathrm{NH}_{2}, \mathrm{CONH}_{2}, \mathrm{CONH}, \mathrm{SO}_{3} \mathrm{H}$ and so on) [1]. The PVP (polyvynilpyrrolidone) is a synthetic polymer, water-soluble as well as in many organic solvent; due to its biocompatibility PVP is one of the more broadly studied hydrophilic materials. PVP has been applied in pharmaceuticals, biomedical sciences, food, and cosmetics $[12,13]$. In this research PVP was used as starting material of the synthesis process.

A multitude of techniques have been described for the synthesis of nanomaterials (nano and micro gels) from polymers. Various approaches have been divided into three major techniques: (1) simultaneous cross-linking polymerization, (2) ideal intramolecular cross-linking, and (3) destruction of macro gel networks into nanoscale (conversion of macroscopic gels to nanogels) [2]. In this research ionizing radiation was used as initiator of synthesis process, because the radiation technique has proved to be particularly suitable for obtaining nanogel for biomedical use, it being our future application. The main factors that contribute to the success of these technologies are: water as a human-and environment-friendly solvent, lack of any potentially harmful chemicals (monomers, initiators, crosslinking agents, etc.), simple production schemes, parallel synthesis and sterilization of the products in the sealed, final packages, valuable medical properties of the products, broad range of applications, and the possibility of manufacturing "smart" gels reacting to temperature, $\mathrm{pH}$, light, etc. [14]. The synthesis of PVP nanogels by gamma radiation for their evaluation as drug delivery system or as potential pharmaceutical carriers was the aim of this paper. Also, the PVP concentration influence on nanogel formation was evaluated. The SEM (scanning electron microscopy), ATR (attenuate total reflection spectroscopy), DLS (dynamic light scattering), and viscosimetry were used as characterization techniques.

\section{Materials and Methods}

\subsection{Materials}

The chemicals used were mostly reagent with high analytical grade and used as received (Sigma-Aldrich) without further treatment. PVP, type K-90 (> 99.99\%). Diluted solutions were prepared with water purified by distillation and in order to remove any dust particles and/or polymer aggregates, solutions were filtered subsequently through filters of 0.45 and 0.22 $\mu \mathrm{m}$ pore size respectively. The $\mathrm{pH}$ and conductivity of the solutions were found to be $8.02 \pm 0.05$ and $\mu=2.4$ $\mu \mathrm{S} / \mathrm{cm} 0.5 \%$ of measured value.

\subsection{Synthesis of PVP Nanogels}

The nanogel was prepared by ideal intramolecular crosslinking method [14] and using gamma radiation as initiator. Experiments were performed using argon saturated solution of PVP (0.1-1\%). Previously the samples were filtered. Gamma irradiation was carried out with a panorama ${ }^{60} \mathrm{Co}$ source at dose of $15 \mathrm{kGy}$ and at room temperature. The irradiation process was monitored by Fricke dosimetry and film dosimeters using Perpex. Irradiation times were determined by calculation CaliPMMA platform, developed at the Laboratory of High Dose Dosimetry Centre for Technological Applications and Nuclear Development (CEADEN) [15] (Fig. 1). 


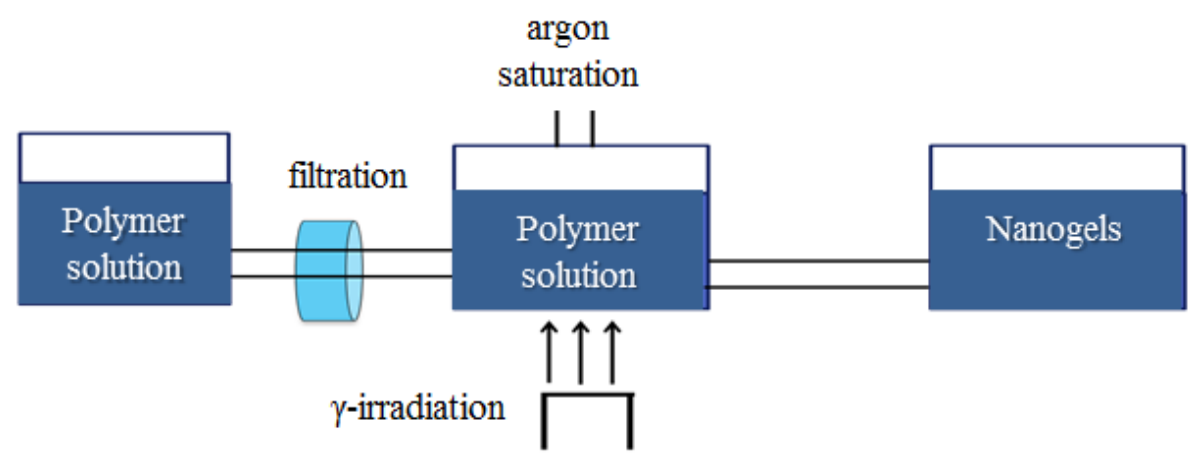

Fig. 1 Scheme of the experimental set-up.

Table 1 Value of the reduced viscosity of unirradiated PVP solutions.

\begin{tabular}{llllll}
\hline PVP concentration $(\mathrm{g} / \mathrm{mL})$ & 0.001 & 0.0025 & 0.005 & 0.0075 & 0.01 \\
\hline Reduced viscosity & 114.21 & 118.75 & 128.38 & 139.74 & 142.00 \\
\hline
\end{tabular}

\subsection{Characterization Techniques}

\subsubsection{Viscosity Measurement}

According to method described in ISO 1628-1: 1998 (E) [16] and NC 30-13 [17] Norms; the measurement of viscosity was executed to PVP aqueous solutions before and after irradiation procedure, the viscosimetric study was carried out by Ubbelhode viscometer. From obtained measurements, reduced viscosity of PVP samples was calculated.

\subsubsection{DLS Measurement}

The molecular weight $\left(M_{w}\right)$, hydrodynamic radius $\left(R_{h}\right)$, and concentration of intermolecular crosslinks [intermolecular] of unirradiatedand irradiated PVP samples were performanced using a particle analyser (DelsaTMNano).

Particularly the [intermolecular] is calculated using Eq. (1):

$$
\text { [intermolecular }]=0,5\left(\frac{1}{M_{w 0}}-\frac{1}{M_{w}}\right) C_{\text {polymer }}
$$

where, $M_{w 0}, M_{w}$ denote the molecular weight before and after irradiation and $C_{\text {polymer }}$ is the polymer concentration [10].

\subsubsection{ATR}

By ATR the chemical structure of unirradiated and irradiated samples of PVP were studied. Sample drops, were deposited onto diamond crystal and then analysis was performed. The study was developed with a
Perkin Elmer Spectrum One spectrometer (ATR) in reflectance mode, with 20 scans and a resolution of 2 $\mathrm{cm}^{-1}$ in the range of $250-4,000 \mathrm{~cm}^{-1}$, at room temperature.

\subsubsection{Morphology by SEM}

The morphology of produced particles was investigated by an SEM (JEOL JSM-6390LV), operated with an accelerating voltage of $0.5-30 \mathrm{kV}$. The amplification was from $\mathrm{X}$ until $300.00 \mathrm{X}$. The analysis was performed employing a (W) filament.

\section{Results and Discussion}

\subsection{Analysis of the Viscosity}

From the flow time measurement of unirradiated solutions was calculated the reduced viscosity for each PVP concentration (Table 1).

When the polymeric solutions were subjected to gamma irradiation, the solutions at $0.5 \%, 0.75 \%$ and $1 \%$ of PVP became crosslinked into a gel structure which is swollen in the initial solvent (water). This transformation from a viscous fluid to a crosslinked gel can be determined by visual inspection of the irradiated solutions [18] (Fig. 2). Whereas, the solutions at $0.1 \%$ and $0.25 \%$ of PVP continue as viscous fluid.

When comparing the reduced viscosity value of unirradiated and irradiated PVP solutions ( 0.1 and $0.25 \%$ ) 

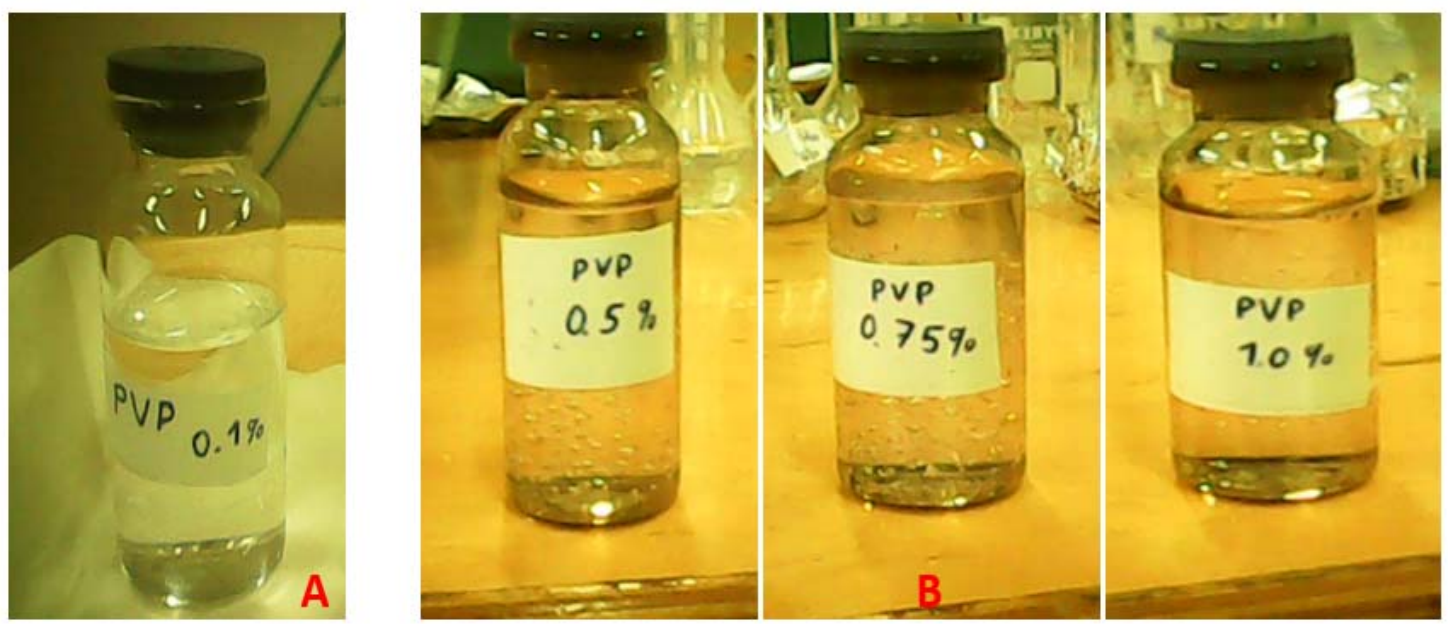

Fig. 2 Comparison of PVP solutions appearance: (a) unirradiated solution and (b) irradiated solutions at 15 kGy.

Table 2 Value of the reduced viscosity of irradiated PVP solutions at $15 \mathrm{kGy}$.

\begin{tabular}{lll}
\hline PVP concentration $(\mathrm{g} / \mathrm{mL})$ & 0.001 & 0.0025 \\
\hline Reduced viscosity & 14.84 & 29.91 \\
\hline
\end{tabular}

Table 3 Value of the $R_{h}, M_{w}$, and [intermolecular] in PVP solutions at $15 \mathrm{kGy}$.

\begin{tabular}{llll}
\hline Concentration $(\%)$ & $\mathrm{Rh}(\mathrm{nm})$ & $\mathrm{Mw}(\mathrm{Da})$ & [intermolecular] $(\mathrm{mol} / \mathrm{L})$ \\
\hline 0.1 & 18.49 & $3.48 \mathrm{E}+06$ & $2.73 \mathrm{E}-07$ \\
0.25 & 19.44 & $4.32 \mathrm{E}+06$ & $7.53 \mathrm{E}-07$ \\
\hline
\end{tabular}

is observed, that reduced viscosity considerably decreases with respect to obtained value for unirradiated solutions (Table 2). It indicates the possible formation of nanogels. Because, as nanogels particles are more compact than the spiral form of its linear polymer, the viscosity of the nanogels is smaller than linear macromolecules at the same concentration.

\subsection{Analysis of the $R_{h}, M_{w}$, and [intramolecular] by $D L S$}

By using the data of DLS it is possible to explain the changes of the crosslinked macromolecule dimension. Results are shown in Table 3.

The hydrodynamic radius and molecular weight of the PVP solution prior to irradiating is $25.26 \mathrm{~nm}$ and 1.2E+06 Da, respectively. The hydrodynamic radius declines when the dimension of internally crosslinked macromolecule decreases and it describes the macromolecule dimension more directly than viscosity data [18]. At 0.1 and $0.25 \%$ PVP concentration the $R_{h}$ value decreases, suggesting intramolecular recombination. This is an expected effect for the process of coil shrinkage resulting from intramolecular crosslinking.

The $M_{w}$ increases in both cases, indicating some contribution of intermolecular recombination. Although it is more pronounced in the solution of $0.25 \%$ PVP due to the macromolecules in solution are closer of the other ones facilitating the intermolecular crosslinking and it is corroborated with [intermolecular crosslinks] result. Finally, the macromolecules mainly crosslink intramolecularly and form nanogels.

\subsection{Analysis of the Chemical Structure}

In order to better realize the changes in chemical structure that take place as a function of the PVP concentration employed, the ATR spectra of irradiated PVP solutions were measured. The vibrational bands of the carbonyl group did not evidence considerable 
changes in both solutions. Whereas, vibrational bands to associated hydroxyl group show intensity changes indicating solute-solute associations, which are stronger to high concentration (Fig. 3).

\subsection{Analysis of the Particles Morphology}

The SEM image shows of polymeric samples at different PVP concentration. In both images it is observed that the obtained nanogel has a mainly elliptical shape with different particles size. This wide polydispersity index is due to that the nanogel particles are in solid state and therefore the particles tend to agglomerated. Also in the $0.25 \%$ PVP solution this effect is more pronounced than in $0.1 \%$ PVP solution due to the intermolecular recombination contribution and it allows micro gel formation (Fig. 4). This result corroborates the obtained results by DLS. It is important to say that due to the agglomeration effect of nanogel particles in solid stated, their size evaluated by SEM is not totally reliable, we suggest for using the TEM (transmission electron microscopy) achieving major accuracy result.

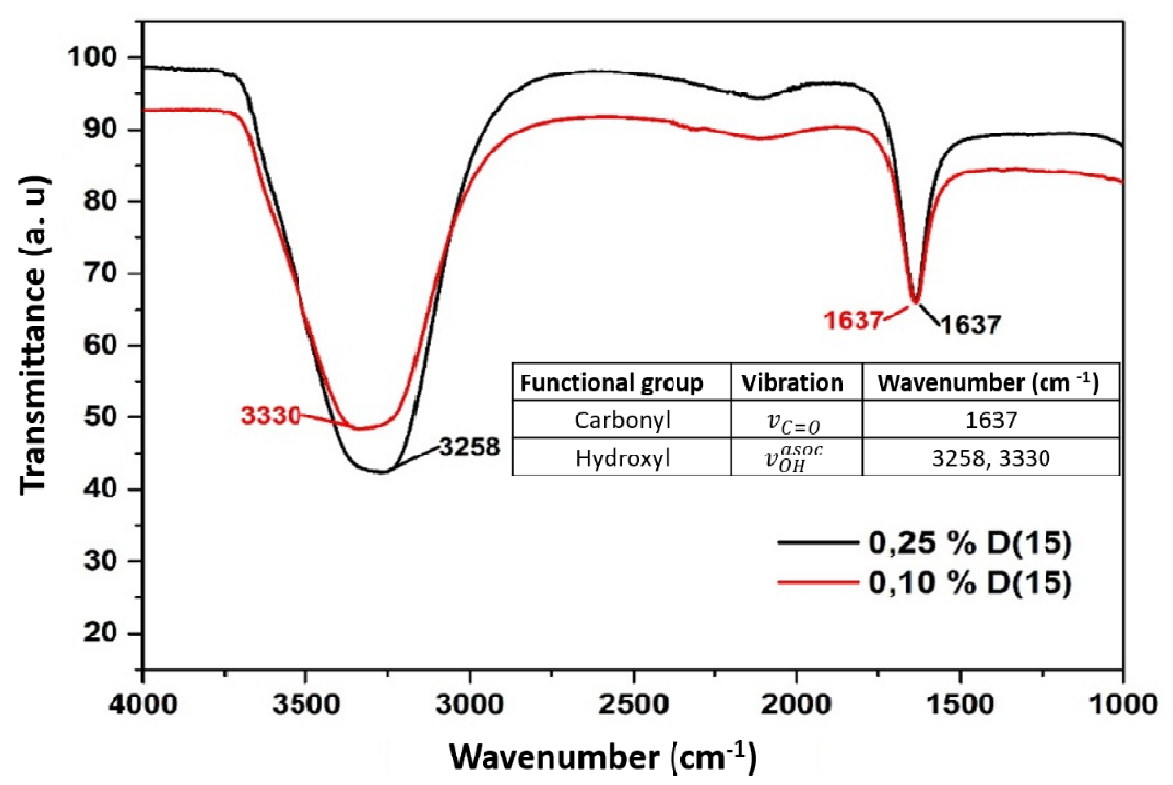

Fig. 3 Influence of PVP concentration on ATR spectra of aqueous PVP solutions at room temperature and 15 kGy.
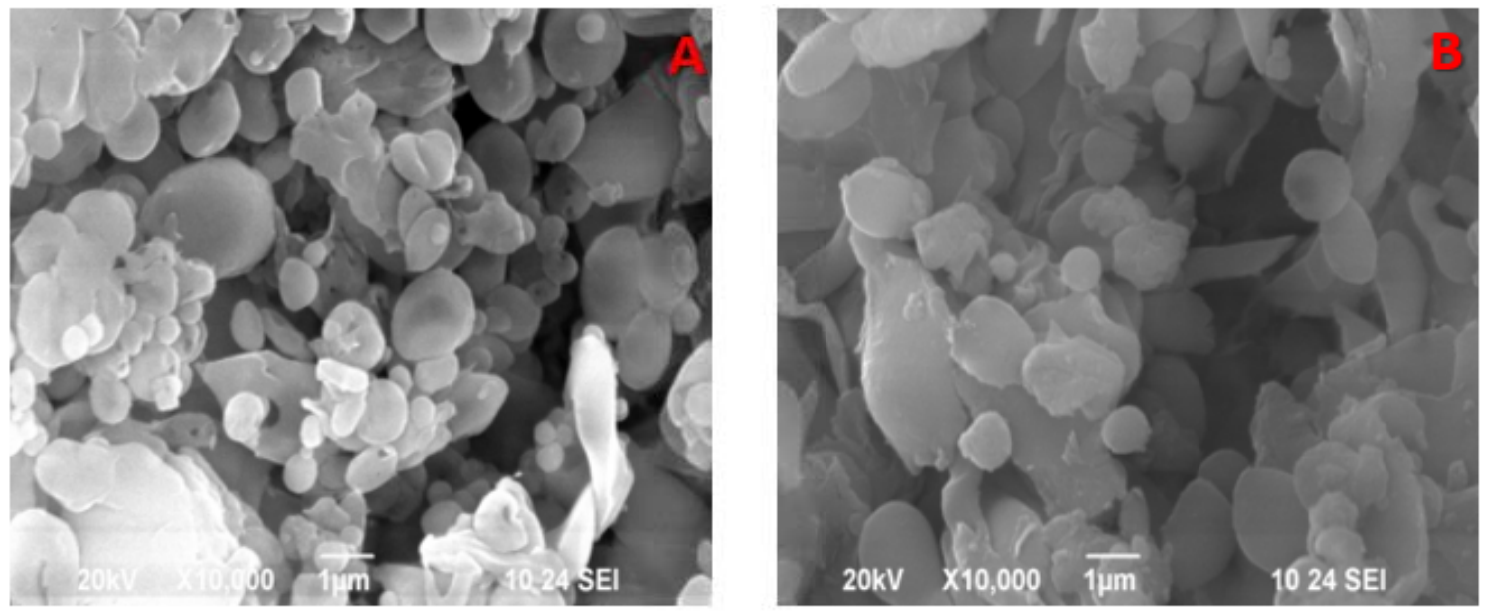

Fig. 4 SEM image of PVP nanogels: (a) $0.1 \%$ concentration and (b) $0.25 \%$ concentration at dose of $15 \mathrm{kGy}$. 


\section{Conclusions}

Nano and micro gel particles of polyvynilpyrrolidone were synthesized by gamma radiation. The synthesis process started from biocompatible polymer without harmful monomers, initiators or crosslinking agents. The PVP concentration has a considerable influence on morphology, chemical structure and crosslinking reactions at a single dose value for obtained product. The nanogels have an elliptical shape forming nanoparticles in solution. In the near future, they could have an extensive field of applications due to their physicochemical properties.

\section{Acknowledgments}

We want to thank to CICATA-Legaria and CNyN in México, CEADEN, InSTEC, CQB and CIGB in Cuba for the support.

\section{References}

[1] Ulanski, P. Y., and Rosiak, J. M. 2004. "Polymeric Nano/Microgels." Encyclopedia of Nanoscience and Nanotechnology.

[2] Labhasetwar, V., and Leslie-Pelecky, D. L. 2007. "Biomedical Applications of Nanotechnology." Nanogels: Chemistry to Drug Delivery: 131-72.

[3] Rosiak, J. M. 1991. Radiation Effects on Polymers, edited by Clough, R. C., and Shalaby, S. W. ACS Book Series Vol. 475. Washington, DC: Am. Chem. Soc., 271.
[4] Hirasa, O. 1993. "Research Trends of Stimuli-Responsive Polymer Hydrogels in Japan.” J. Intelligent Mater. Syst. Struct. 4 (538).

[5] Pelton, R. 2000. "Temperature-Sensitive Aqueous Microgels.” Adv. Colloid Interface Sci. 85 (1).

[6] Rosiak, J. M., Rucinska-Rybus, A., and Pekala, W. 1989. U.S. Patent 4,871,490.

[7] Hille, H.-D., Neis, S., and Muller, H. 1999. U.S. Patent $5,977,258$.

[8] Roth, M., Tang, Q., and Eldin, S. H. 1999. U.S. Patent $5,994,475$.

[9] Tranchant, J.-F., Riess, H.-G., and Meybeck, A. 2001. U.S. Patent 6,280,713.

[10] Sultana, F., Md. Imran-Ul-Haque, M., Arafat, M., and Sharmin, S. 2013. "An Overview of Nanogel Drug Delivery System.” J App Pharm Sci 3.

[11] Gogotsi, Y. 2006. Nanomaterilas Hanbook. New York, United Stated of America: CRC Press Taylor \& Francis Group.

[12] Haaf, F., Sanner, A., and Straub, F. 1985. "Polymers of N-Vinylpyrrolidone: Synthesis, Characterization and Uses." Polym, J. 17: 143-52.

[13] Hoffman, A. S. 2002. "Hidrogels for Biomedical Applications." Adv. Drug Delivery Rev. 54 (3).

[14] Rosiak, J. M., Ulanski, P., Kujawa, P., Janik, I., Stasica, P., Kadlubowski, S., and Kozicki, M. 2005. "Radiation-Induced Transformations of Polymers in Aqueous Solutions." Eurasian Chem Tech 7: 225-50.

[15] Plasencia, M. 2005. Registrants CENDA \#1853, 1-2.

[16] ISO Norm s f 1998 ISO 1628-1.

[17] Cuban Norm s f NC 30-13, 82.

[18] Ulanski, P., and Rosiak, J. M. 1999. "The Use of Radiation Technique in the Synthesis of Polymeric Nanogels." Nucl. Instrum. Methods B 151: 356-60. 